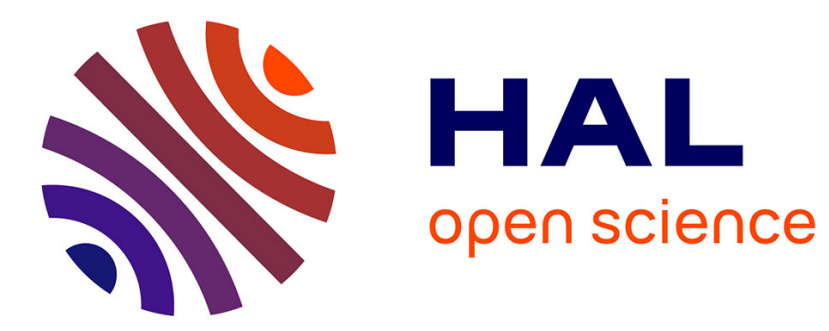

\title{
Les sous-unités gluténines de haut poids moléculaire des blés tendres et des blés durs cultivés en France
}

\author{
Gerard G. Branlard, Annick Le Blanc
}

\section{To cite this version:}

Gerard G. Branlard, Annick Le Blanc. Les sous-unités gluténines de haut poids moléculaire des blés tendres et des blés durs cultivés en France. Agronomie, 1985, 5 (6), pp.467-477. hal-00884773

\section{HAL Id: hal-00884773 \\ https://hal.science/hal-00884773}

Submitted on 1 Jan 1985

HAL is a multi-disciplinary open access archive for the deposit and dissemination of scientific research documents, whether they are published or not. The documents may come from teaching and research institutions in France or abroad, or from public or private research centers.
L'archive ouverte pluridisciplinaire $\mathbf{H A L}$, est destinée au dépôt et à la diffusion de documents scientifiques de niveau recherche, publiés ou non, émanant des établissements d'enseignement et de recherche français ou étrangers, des laboratoires publics ou privés. 


\title{
Les sous-unités gluténines de haut poids molé- culaire des blés tendres et des blés durs cultivés
}

en France

\author{
Gérard BRANLARD \& Annick LE BLANC $\left({ }^{*}\right)$ \\ I.N.R.A., Station d'Amélioration des Plantes, F 63039 Clermont-Ferrand \\ (*) Laboratoire de Physiologie Végétale, Université des Sciences et Techniques de Lille I, F 59655 Villeneuve \\ $d^{\prime}$ Ascq Cedex
}

\begin{abstract}
L'observation des sous-unités gluténines de haut poids moléculaire a été faite par électrophorèse en milieu SDS sur 195 blés tendres, pour la plupart d'origine française, et 38 blés durs. On observe 18 bandes de mobilité différente dans l'ensemble des blés tendres et 14 chez les blés durs. Une nouvelle sous-unité a été répertoriée et sa mobilité se situe entre celle des bandes 10 et 12 . Cette nouvelle bande, appelée 11 , vraisemblablement codée par un gène situé sur $1 \mathrm{DL}$ porte à 24 le nombre de sous-unités gluténines de haut poids moléculaire connues à ce jour. 38 types de diagrammes différents ont été observés chez les blés tendres ; 9 types ont été rencontrés chez les blés durs. Les diagrammes de l'ensemble des blés tendres et durs analysés sont donnés. On constate que l'analyse des gluténines peut s'avérer très intéressante pour distinguer les blés non séparés par l'électrophorèse de leurs gliadines sur gel d'amidon. L'étude de la diversité des blés à l'aide de ces sous-unités gluténines nous a permis de constater (1) que les types de diagrammes rencontrés sont peu nombreux par rapport à l'ensemble potentiellement observable, (2) que les fréquences alléliques chez les blés tendres français sont notablement différentes de celles rencontrées sur une collection mondiale et (3) de confirmer l'augmentation de la ressemblance des blés français entre eux au cours des 20 dernières années.
\end{abstract}

Mots clés additionnels : Triticum aestivum, Triticum durum, protéines, polymorphisme, identification variétale, diversité génétique.

Observation of high molecular weight (HMW) glutenin subunits has been carried out by SDS PAGE for 195 bread wheats, mainly of French origin, and 38 durum wheats. For the bread wheats and the durum wheats respectively, 18 and 14 bands with different mobility were observed. A new subunit migrating between the band 10 and 12 is described. This new band, called 11 , probably coded by a gene located on $1 \mathrm{DL}$, brings to 24 the total number of the HMW subunits known at present. 38 different patterns were observed among the whole set of bread wheats, and 9 among the durum wheats. Patterns for all the bread and durum wheats are given. SDS-PAGE of glutenins could prove very useful for the distinction of wheats which have the same gliadin pattern on starch gel. Study of these subunits led us to the following conclusions on the diversity of wheat : (1) the observed patterns were not very numerous compared with those potentially observable, (2) allelic frequencies of the French bread wheats were markedly different from those observed in a world collection, (3) French bread wheat varieties have become increasingly similar over the last 20 years.

Additional key words : Triticum aestivum, Triticum durum, proteins, polymorphism, varietal identification, genetic diversity.

\section{INTRODUCTION}

Les protéines de réserve des blés tendres (Triticum aestivum) et des blés durs ( $T$. durum) sont formées de 2 classes définies notamment par des critères de solubilité : les gliadines et les gluténines. Ces protéines représentent chez ces 2 espèces plus de 75 p. 100 des protéines du grain (MOSSE, 1968). L'étude électrophorétique des gliadines a permis de mettre en évidence leur polymorphisme (FEILLET, 1965; FEILLET \& BOURDET, 1967) et de l'utiliser comme critère de distinction et d'identification variétale (AUTRAN \& BOURDET, 1975). Le polymorphisme des gliadines, beaucoup plus important que celui des gluténines, est 
contrôlé par des gènes situés sur les bras courts des chromosomes 1 et 6 des 3 génomes (WRIGLEY \& SHEPHERD, 1973 ; KASARDA et al., 1976). Les gluténines et plus particulièrement leurs sous-unités de haut poids moléculaire correspondent à des gènes situés sur les bras longs des chromosomes 1A, 1B et 1D (PAYNE et al., 1980). Les gliadines et gluténines formant la majeure partie des protéines du gluten sont aujourd'hui mieux connues par le rôle qu'elles peuvent jouer dans la qualité des blés tendres (PAYNE et al., 1979, $1981 a$; BRANLARD \& ROUSSET, 1980 ; BURNOUF \& BOURIQUET, 1980 ; BRANLARD \& BELLOT, 1983) et des blés durs (DAMIDALX \& FEILLET, 1978 ; AUTRAN, 1981). Ces études nous offrent une meilleure compréhension des bases biochimiques et génétiques de la qualité des blés. Cependant si l'on connaît bien la diversité des gliadines, grâce notamment aux travaux de Autran qui a en outre étudié les gluténines de 80 blés durs (AUTRAN, 1981 ; AUTRAN et al., 1982), aucune étude en revanche ne s'est attachée à décrire l'hétérogénéité des gluténines des blés tendres français. Le polymorphisme des sous-unités des gluténines de haut poids moléculaire a été précisé pour un ensemble de blés provenant d'une large collection mondiale par PAYNE et al. (1981b). La connaissance de ce polymorphisme peut être utile comme complément à l'identification variétale (AUTRAN et al., 1981). La distinction variétale est parfois impossible par l'observation des gliadines seules. L'observation du polymorphisme des gluténines comme complément pouvant être utilisé dans l'identification variétale n'est par le seul objet de cette étude. En effet l'analyse des gluténines d'une large collection de blés peut nous apporter d'utiles informations sur la diversité des spectres au sein de chacune des espèces. Observe-t-on de nouvelles sous-unités de gluténines par rapport à celles répertoriées par PAYNE sur une large collection mondiale? Ces protéines qui, comme les gliadines, constituent des marqueurs biochimiques, peuvent nous fournir des informations sur l'évolution de la diversité des blés au cours des cycles successifs de la création variétale. Ces questions ont été abordées par l'analyse électrophorétique des sous-unités gluténines d'une large collection de blés tendres et d'un nombre plus restreint de blés durs.

\section{MATÉRIEL ET MÉTHODES}

\section{A. Matériel végétal}

La collection de blés tendres analysés est relativement importante; elle comprend 195 cultivars. Ces 195 blés ont, pour la majorité d'entre eux, été sélectionnés Bora France et inscrits au Catalogue officiel des espèces entre variétés en 1909 et 1983 . Dans cette collection nous trouvons respectivement les 104 et 22 blés d'hiver et de printemps qui sont présents au Catalogue officiel en 1983. La liste des blés tendres est donnée au tableau 2.

Le polymorphisme des gluténines de blés durs a également été étudié. 38 blés durs pour la plupart d'origine française ont été analysés. Parmi ceux-ci 24 sont présents en 1983 au Catalogue. La liste des blés durs est donnée au tableau 3.
Les blés tendres et durs proviennent des Stations I.N.R.A. de Clermont-Ferrand, Montpellier et des Etablissements MOMONT-HENNETTE (Mons-en-Pévèle, Nord). Les variations propres aux lieux et conditions de culture ne modifient pas qualitativement le polymorphisme des sous-unités gluténines. La quantité de chacune de ces protéines, synthétisée au niveau de l'albumen triploïde, est vraisemblablement influencée par les facteurs agro-climatiques comme le sont les gliadines (BRANLARD \& TRIBOI, 1983). Puisque ces sous-unités demeurent qualitativement inchangées par électrophorèse sur gel d'acrylamide en milieu SDS à PH 8,8, l'analyse des cultivars a été réalisée sur les grains pris individuellement et non sur farine. Pour chaque cultivar, 3 grains au minimum ont été analysés.

\section{B. Méthodes}

\section{Electrophorèse des gluténines}

\section{a) Extraction des gluténines}

L'extraction se fait à partir d'un grain préalablement pesé, puis écrasé au mortier. Un demi-grain (la partie albumen) est suffisant si l'on veut conserver l'embryon. A cette masse $m$ de grain broyé placé dans un tube Eppendorf de $2 \mathrm{ml}$ on ajoute la solution d'extraction à raison de $(\mathrm{m} \times 24,5) \mu \mathrm{l} ; \mathrm{m}$ est exprimé en $\mathrm{mg}$. Cette solution d'extraction est constituée d'un tampon Tris-HCl à pH 6,8 $(0,0938 \mathrm{M}$ de Tris $)$, contenant 3 p. $100(\mathrm{p} / \mathrm{v})$ de Sodium Dodécyl Sulfate (SDS), 7,5 p. $100(\mathrm{v} / \mathrm{v})$ de 2-mercapto-éthanol et du bleu de bromophénol environ $0,01 \mathrm{p} .100(\mathrm{p} / \mathrm{v})$. Le mélange grain broyé + solution d'extraction, une fois homogénéisé, est laissé à température ambiante pendant $2 \mathrm{~h}$ puis placé dans un bain-marie bouillant pendant $5 \mathrm{mn}$ afin de parfaire l'extraction des protéines et leur réduction en sous-unités. Après centrifugation à $10000 \mathrm{~g}$ pendant $5 \mathrm{mn}, 15 \mu \mathrm{l}$ ou $40 \mu \mathrm{l}$ de surnageant seront utilisés selon qu'on dispose d'un gel d'électrophorèse de 1,5 ou $3 \mathrm{~mm}$ d'épaisseur. Les extraits peuvent être conservés au congélateur.

\section{b) Electrophorèse des gluténines}

La technique d'électrophorèse utilisée est celle proposée par LAEMMLI (1970). Le support d'électrophorèse est formé d'un gel de concentration (Stacking gel) contenant 5 p. $100(\mathrm{p} / \mathrm{v})$ d'acrylamide, 0,09 p. 100 $(\mathrm{p} / \mathrm{v})$ de méthylène bis acrylamide (Bis) tamponné à pH 6,8 et d'un gel de séparation (Separating gel) contenant 10 p. $100(\mathrm{p} / \mathrm{v})$ d'acrylamide, 0,3 p. $100(\mathrm{p} / \mathrm{v})$ de Bis tamponné à $\mathrm{pH} 8,8$. Ces 2 gels contiennent également 0,1 p. $100(\mathrm{p} / \mathrm{v})$ de SDS et sont polymérisés en présence de TEMED et d'ammonium persulfate. Pour séparer les bandes 2 et $2^{*}$ confondues sur le gel à 10 p. 100 d'acrylamide, on réalise un gel à 7 p. 100 d'acrylamide.

Le dépôt des protéines une fois effectué, le gel qui a une dimension de $160 \times 160 \times 1,5 \mathrm{~mm}$ est soumis à une tension constante de $60 \mathrm{~V}$ pendant environ $18 \mathrm{~h}$. Les protéines chargées négativement migrent vers l'anode et sont séparées selon leur encombrement moléculaire. La migration est arrêtée lorsque le colorant (bleu de bromophénol) quitte le gel. 


\section{c) Coloration des gels}

Les protéines sont colorées au bleu de Coomassie R 250 à 0,1 p. 100 dans une solution eau-isopropanolacide acétique (65:25:10, v/v) pendant $2 \mathrm{~h}$. Les gels sont ensuite décolorés dans de l'acide acétique à 10 p. 100 pendant 1 à $2 \mathrm{~h}$ et placés dans l'eau pendant toute une nuit. Le gel peut ensuite être photographié, séché ou conservé dans un sachet plastique thermosoudé.

\section{Calcul des distances intergénotypiques}

Les sous-unités de haut poids moléculaire des gluténines constituent des marqueurs biochimiques qui, comme les gliadines, peuvent être utilisés dans le but de décrire la diversité existant entre génotypes. Le calcul de la distance entre 2 blés a été réalisé en comparant les 2 diagrammes $i$ et $i^{\prime}$ pour chacune des $n$ bandes possibles. Le calcul du moment centré d'ordre 2 a été effectué pour estimer la distance entre cultivars.

$$
d^{2}\left(i, i^{\prime}\right)=\sum_{j=1}^{n} \frac{1}{C \cdot j}\left(\frac{C_{i j}}{C_{i}}-\frac{C_{i^{\prime} j}}{C_{i^{\prime}}}\right)^{2}
$$

où $\mathrm{Cij}$ vaut 1 si la bande $\mathrm{j}$ est observée chez l'individu $i$, et 0 si non.
$\mathrm{Ci}$ (ou $\mathrm{Ci}^{\prime}$ ) représente le nombre moyen de bandes observées chez l'individu i (ou i').

C.j représente la fréquence moyenne de la bande $\mathrm{j}$ dans l'échantillon soumis à l'analyse.

C'est la racine carrée de (1) qui a été prise en compte pour le calcul de la distance entre génotypes. L'évolution au cours du temps de la diversité génétique a été étudiée en calculant les distances entre les $\mathrm{N} 1$ cultivars inscrits au cours d'une période 1 et les N2 cultivars d'une période 2 . Sur les N1 $\times$ N2 distances obtenues on a calculé la moyenne et l'écart-type.

Pour le calcul de la distance génétique entre cultivars, il a été procédé de même en tenant compte cette fois-ci non pas des bandes mais des allèles qui codent pour leur synthèse.

\section{RÉSULTATS ET DISCUSSION}

\section{A. Les différents types de bandes observés}

\section{Cas des blés tendres}

L'analyse des diagrammes électrophorétiques des 195 blés tendres nous a permis de distinguer 18 types de mobilités différentes. Ces 18 bandes différentes ne

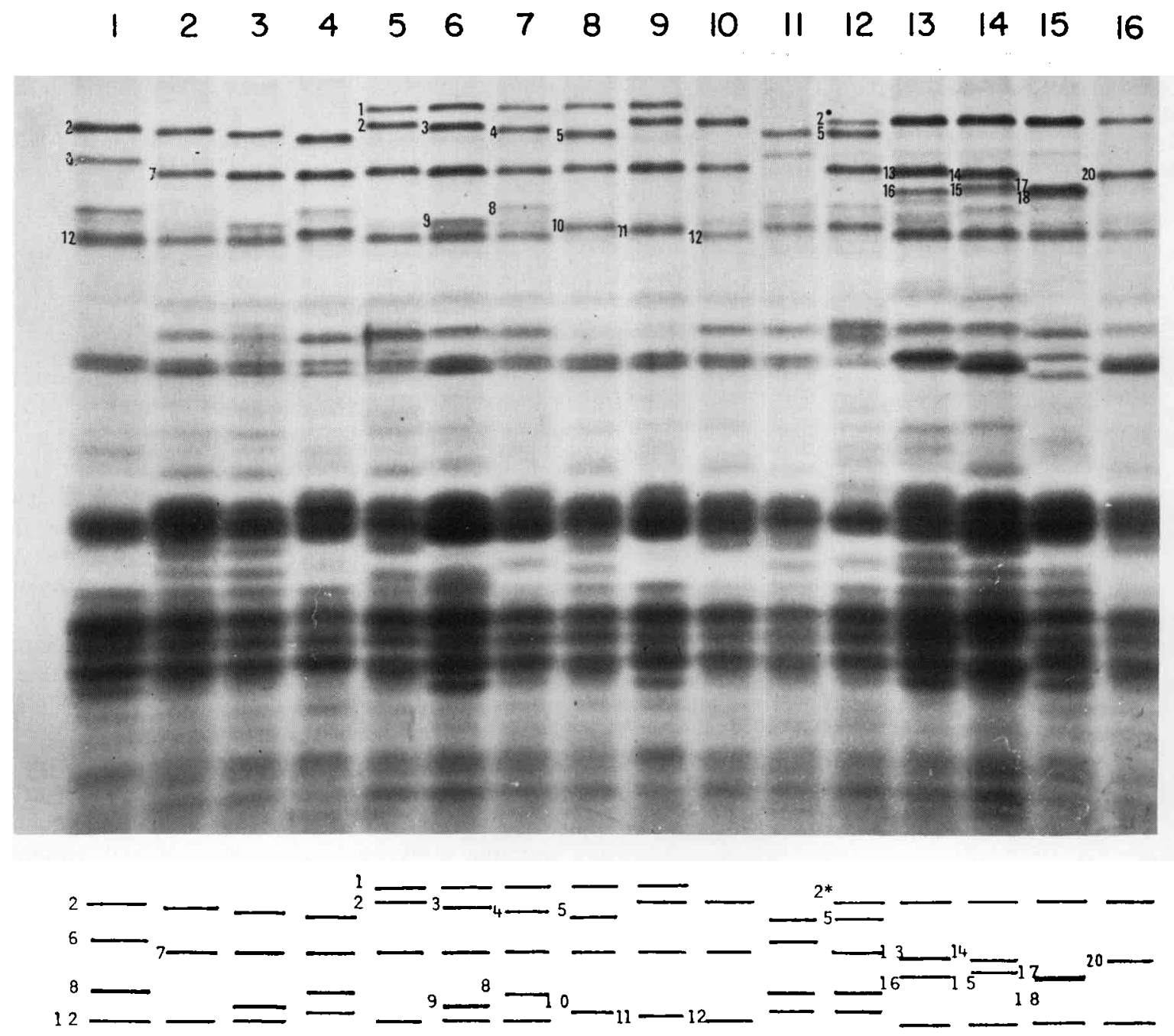

Figure 1

Polymorphisme des sous-unités gluténines de haut poids moléculaire de 16 cultivars.
Polymorphism of HMW glutenin subunits of 16 cultivars : 1 : Clément, 2: Hobbit, 3: Allegro, 4: Eylau, 5: Marignan, 6: Rock, 7: Gamin, 8: Ducat, 9: Flinor, 10: Hardi, 11: Eloi, $12:$ Cargo, $13:$ Atlas 66, $14:$ Sappo, $15:$ Prinqual, 16: Primépi. 
recouvrent que les sous-unités gluténines de haut poids moléculaire compris entre 75000 et 130000 .

Nous donnons à la figure 1 les principaux types rencontrés sur la collection de blés tendres à laquelle plusieurs types de blés d'origine étrangère ont été ajoutés. Sur les 20 bandes de mobilités différentes qui sont présentes sur ces diagrammes 19 sont nettement distinguables. Selon la nomenclature de PAYNE \& LAWRENCE (1983) que nous avons adoptée (tabl. 1), ces 20 bandes sont les suivantes par ordre décroissant de poids moléculaire : $1,2,2^{*}, 3,4,5,6,7,13,14,20$, $15,16,17,18,8,9,10(11)$ et 12 . La bande 18 est généralement difficilement distinguable de la bande 17 lorsqu'on réalise la séparation sur un gel à 10 p. 100 d'acrylamide. C'est le cas de la variété "Prinqual » (diagramme 15 de la photo 1 ) dont le spectre est $2,2^{*}$, $17,18,12$. Une bande n'est pas distinguable sur ce type de gel lorsqu'une autre bande de mobilité voisine est présente. Il s'agit de la bande $2^{*}$ qui se confond avec la 2 lorsque celle-ci est.présente. Ces deux bandes $2^{*}$ et 2 sont respectivement sous le contrôle d'un gène situé sur le bras long du chromosome $1 \mathrm{~A}$ et 1D (PAYNE et al., 1980). La bande $2^{*}$ est présente seule chez «Cargo » (diagramme 12) et associée à la bande 2 chez «Hardi », «Sappo » et «Prinqual» (diagrammes 10,14 et 15 respectivement).

TABLEAU 1

Nomenclature des sous-unités gluténines de haut poids moléculaire classées par mobilité croissante: I, II, III respectivement selon PAYNE \& LAWRENCE (1983), BURNOUF \& BOURIQUET (1980), MOONEN et al. (1982).

Nomenclature of HMW glutenin subunits in order of increasing mobility, according to I: PAYNE \& LAWRENCE (1983), II : BURNOUI \& BOURIQUET (1980), III : MOONEN et al. (1982).

\begin{tabular}{|c|c|c|}
\hline I & II & III \\
\hline \multicolumn{3}{|l|}{2,2} \\
\hline 1 & 1 & 1 \\
\hline 2 & 2 & 2 \\
\hline $2^{*}$ & 3 & \\
\hline \multicolumn{3}{|l|}{3} \\
\hline \multicolumn{3}{|l|}{4} \\
\hline 5 & $3^{\prime \prime}$ & 3 \\
\hline 6 & 4 & 4 \\
\hline 7 & 5 & 5 \\
\hline 13 & & 6 \\
\hline \multicolumn{3}{|l|}{14} \\
\hline \multicolumn{3}{|l|}{20} \\
\hline \multicolumn{3}{|l|}{19} \\
\hline \multicolumn{3}{|l|}{15} \\
\hline 16 & & 7 \\
\hline \multicolumn{3}{|l|}{17} \\
\hline \multicolumn{3}{|l|}{18} \\
\hline \multicolumn{3}{|l|}{21} \\
\hline \multicolumn{3}{|l|}{22} \\
\hline 8 & 6 & 8 \\
\hline 9 & $6 "$ & 9 \\
\hline 10 & & 10 \\
\hline 12 & 7 & 11 \\
\hline
\end{tabular}

Pour s'assurer que la bande 2 est seule et non associée à la bande $2^{*}$, on réalise une électrophorèse à 7 p. 100 d'acrylamide en déposant les extraits protéiques des diagrammes pour lesquels le doute subsiste. La séparation est alors nette (voir fig. 2).

La variété «Prestige » possède la bande 19 qui n'est pas rapportée sur la figure 1. Par électrophorèse

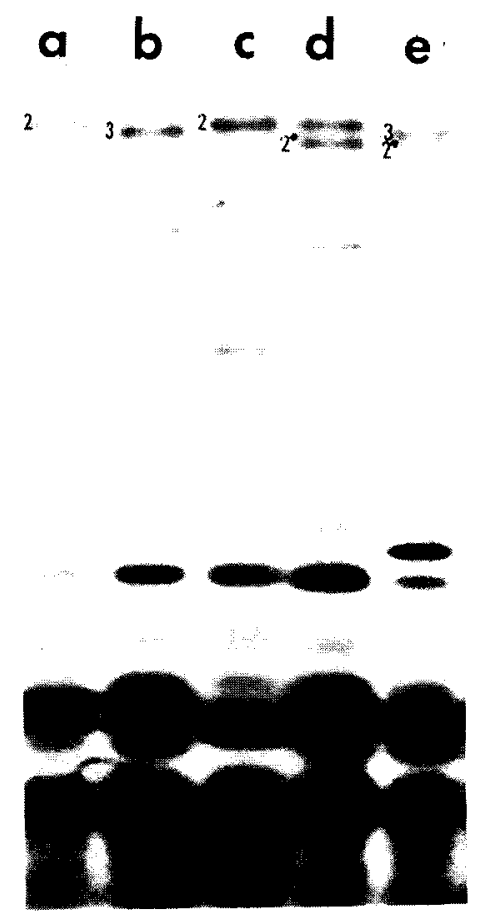

Figure 2

Polymorphisme des sous-unités gluténines de 5 cultivars sur un gel à $7 \%$ d'acrylamide en milieu SDS. Les bandes 2 et $2 *$ sont ainsi séparées.

$7 \%$ polyacrylamide gel electrophoretic patterns (SDS-PAGE) of 5 cultivars. Subunits 2 and $2 *$ were separated in a $7 \%$ gel but not in a $10 \%$ one.

$a$ : Frondoso, $b:$ Hobbit, $c:$ Clément, $d:$ Sappo, e : lignée $F_{6}$ issue d'un croisement entre Courtot $(2,2 *, 7,12)$ et Corin $(3,6,8,12)$.

en milieu SDS à $\mathrm{pH} 8,8$ avec un gel à 10 p. 100 d'acrylamide, la mobilité de cette protéine 19 se situe entre les bandes 20 et 15 . Son poids moléculaire est très voisin de celui de ces 2 bandes.

Les bandes que nous n'avons pas rencontrées dans les 195 blés français sont les suivantes : 13, 15, 16, 21 et 22 toutes sous le contrôle de $1 \mathrm{BL}$ et la bande $(2,2)$ qui serait dépendante d'un gène situé sur $1 \mathrm{DL}$. Cette dernière sous-unité a été récemment décelée par PAYNE et al. (1983) chez certains cultivars japonais tels «Danchi » et « Norin 20 ». Les bandes 21 et 22 sont également très rares et présentes chez quelques blés d'origine yougoslave.

En revanche, nous avons observé une bande non répertoriée jusqu'ici (LE BLANC, 1984). Cette bande nouvelle a une mobilité qui se situe entre les bandes 10 et 12 . Généralement ces deux bandes 10 et 12 sont distinguables. Mais les améliorations apportées à la technique d'électrophorèse permettent de les séparer plus nettement encore et d'offrir la possibilité de répertorier une nouvelle bande que nous avons appelée 11 . Nous donnons à la figure 3, quatre des 6 diagrammes des sous-unités gluténines qui présentent cette bande 11. Il s'agit de «Abel », « Bléroy », «Cordial », «Elite ", « Flinor » et " Marly ». Il semble que cette bande proviennent de "Bellevue » qui est parent de «Abel », "Bléroy » et « Elite ». Ce dernier a transmis cette bande 11 à « Cordial», «Flinor » et « Marly » car ces 3 cultivars proviennent respectivement des croisements «Elite» $x$ « Marne », "Elite » $X$ « Poncheau » et « Elite » $x$ " Marne ». 

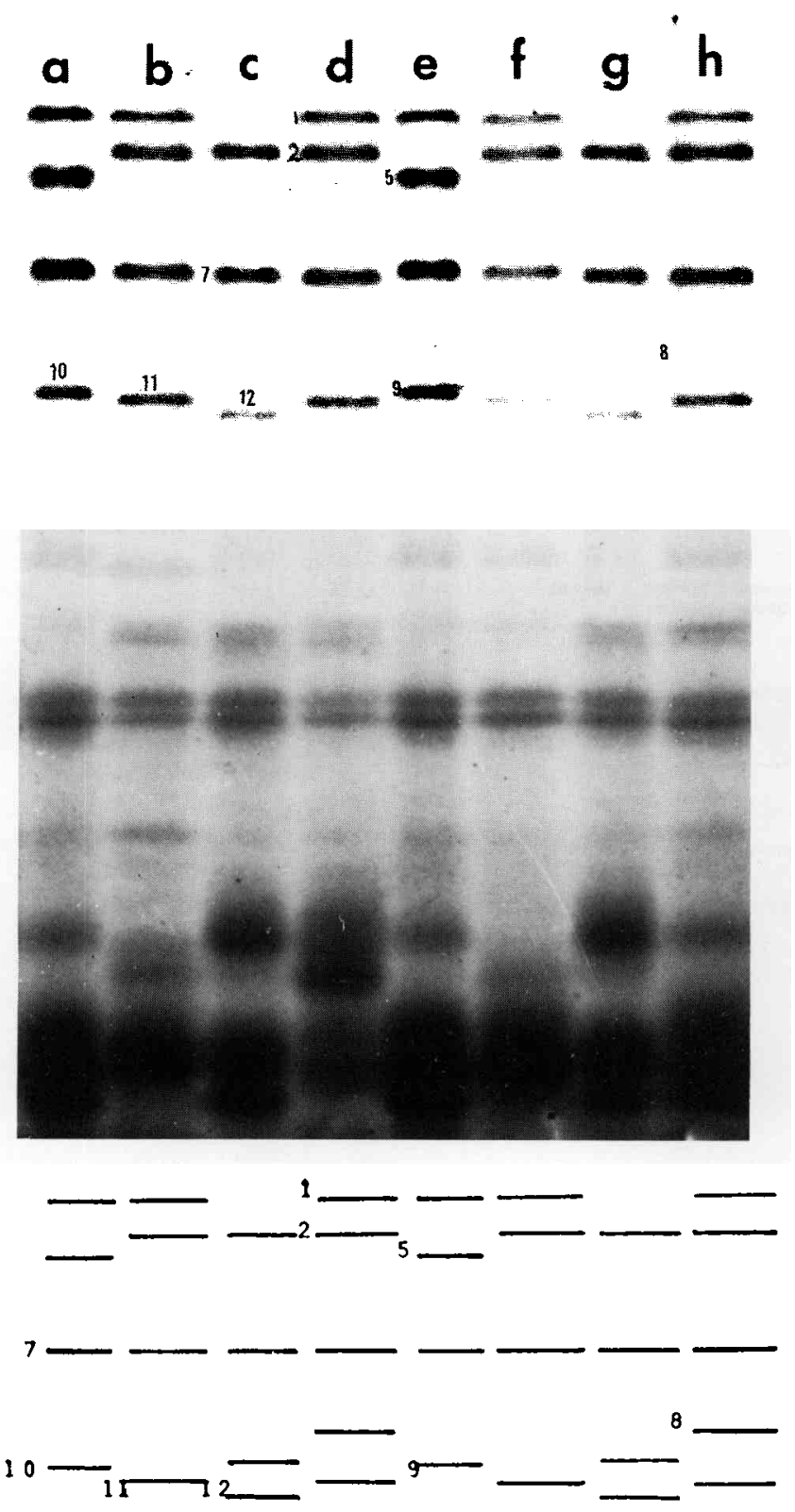

Figure 3

Diagramme des sous-unités gluténines de 6 cultivars dont 4 possèdent la bande 11 . a et $e:$ Ducat ; $b:$ Cordial; $c$ et $g$ : Capitole; $d:$ Elite ; $f:$ Flinor $; h:$ Bleroy.

Polyacrylamide gel electrophoretic patterns of cultivars which show presence or absence of band 1I. Slots $b, d, f$ and h show distinctly this band migrating between band 10 of Ducat (slots $a$ and e) and band 12 of Capitole (slots $c$ and $g$ ) ; $b$ : Cordial ; $d:$ Elite ; $f: F / i-$ nor ; $h$ : Bléroy.

Cette bande 11 est associée à la bande 2. Elle est donc comme cette dernière dépendante d'un gène situé sur le bras long du chromosome 1D. PAYNE \& LAWRENCE (1983) avaient décrit pour «Flinor » un nouveau type d'association entre la bande 2 et la bande 10. Nous montrons ici qu'il ne s'agit pas de la bande 10 mais d'une nouvelle bande que l'on note 11 . Le nombre de bandes des gluténines de haut poids moléculaire est donc à ce jour de 24 et 18 ont été observées sur les 195 blés français.

\section{Remarques sur l'identification des bandes}

1) Dans les conditions habituelles d'électrophorèse un certain nombre de bandes peuvent se dédoubler. Ce phénomène se produit particulièrement pour les bandes 8,9 et plus rarement pour les bandes 10 et 12 . Chaque fois que cela se produit on n'enregistre qu'une seule bande.

2) Certaines bandes peuvent apparaître très faiblement concentrées et généralement plus fines que les 24 autres recensées jusqu'ici. La présence de ces bandes n'est pas systématique et, par conséquent, elles ne sont pas enregistrées. Ce phénomène peut se produire notamment lorsque l'extraction des protéines et/ou leur séparation en sous-unités sont incomplètes. Des modifications (bandes plus floues, apparition de bandes fines) peuvent également se produire lorsque l'extrait est gardé trop longtemps au réfrigérateur.

Une schématisation des diagrammes électrophorétiques a été donnée aux figures 1 et 3.

\section{Cas des blés durs}

Dans ce cas, les bandes qui sont sous la dépendance du chromosome 1 DL sont absentes. Il s'agit des bandes $2,3,4,5,10,11$ et 12 . On observe pour les 38 blés durs analysés 11 types de bandes de mobilités différentes. Ce sont celles qui sont sous la dépendance des chromosomes $1 \mathrm{AL}$ et $1 \mathrm{BL}$, c'est-à-dire les bandes $1,2^{*}, 6,7,13,14,20,19,16,8$ et 9 . A ces 11 bandes s'ajoutent plusieurs bandes généralement faiblement concentrées dont la mobilité est comprise entre celle des bandes 20 et 10 . L'une d'entre elles est de même mobilité et donc de même poids moléculaire apparent que la bande 10 qui est sous le contrôle de 1 DL. D'autres études sont donc nécessaires pour vérifier si cette bande que nous avons notée $10^{\prime}$ est identique à la bande 10 . Si tel était le cas, cela signifierait que le gène de structure de la bande 10 se trouverait sur $1 \mathrm{BL}$. Le chromosome $1 \mathrm{AL}$ semble exclu puisque cette bande $10^{\prime}$ est associée aux bandes 6 et 8 qui sont dépendantes de $1 \mathrm{BL}$. Notons que ce type de duplication de gène a été rencontré chez certaines oméga-gliadines (BRANLARD, 1983).

Pour les blés tendres comme pour les blés durs, ces diverses bandes ne sont pas toutes transmises indépendamment les unes des autres. Certaines correspondent à des séries alléliques, d'autres à des gènes liés. Ces structures génétiques se traduisent par différents types de diagrammes que nous allons détailler pour les blés tendres et durs cultivés en France.

\section{B. Les différents types de diagrammes}

\section{Cas des blés tendres}

Au tableau 2 sont portés les 38 types de diagrammes des sous-unités gluténines observés chez les 195 blés tendres d'hiver et de printemps. L'analyse de ce tableau nous montre que la diversité des sous-unités gluténines est, à elle seule, un très mauvais outil pour identifier les variétés. La prise en compte de bandes appartenant au groupe des sous-unités de poids moléculaire moyen nous aurait vraisemblablement permis d'observer davantage de diagrammes différents (AUTRAN et al., 1981). Sur ces 195 cultivars nous observons en effet 25 diagrammes identiques ayant les bandes 2-7-12 : ensemble que l'on peut noter $25 \mathrm{~d}$. 
TABLEAU 2

Diagrammes des sous-unités gluténines de 195 blés tendres pour la plupart d'origine française. Glutenin subunit patterns of 195 (mainly French) bread wheats.

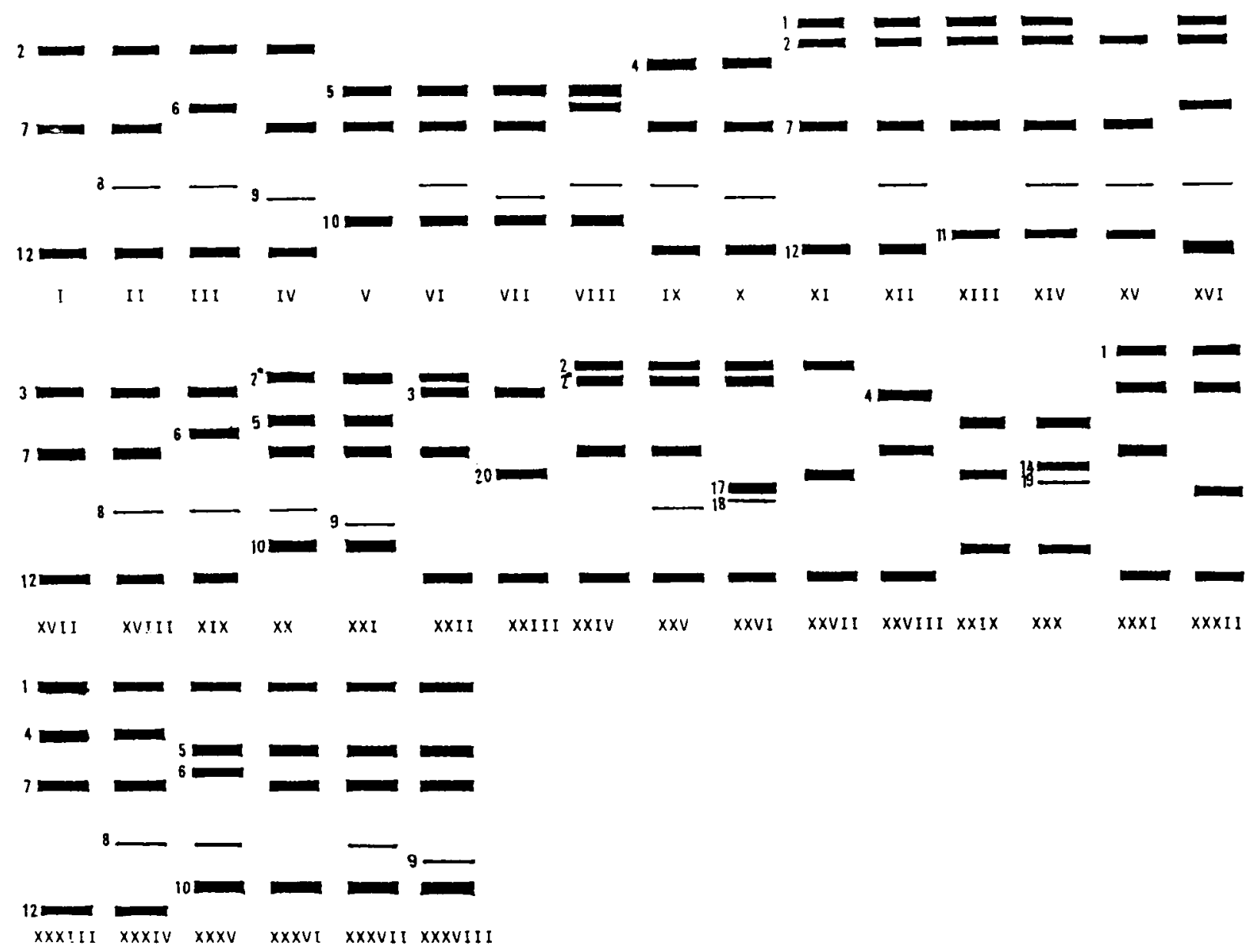

I ; 2, 7, 12 : Agriss, Aquila, Arminda, Arromanches, Braco, Cappelle-D, Carlos, Darius, Decius, Festin, Francest, Heurtebise, Kinsman, Mistral, Noroit, Prieur, Pursang, Remois, Roazon, Rotonde, Rudi, Somme, Tadepi, Thermidor, Trio.

II ; 2, 7, 8, 12 : Albatros, Astral, Athos, Aviso, Axel, Blason, Choisy, Gaillard, Huquin, Magali, Marengo, Moisson, Promesse, Radja, Rendor, Sabre, Scipion.

III ; 2, 6, 8, 12 : Asso, Armada, Beauchamp, Clément, Cocagne, Essor, Heima, Horace, Joss, Magister, Maris-Huntsman, Riol.

IV ; 2, 7, 9, 12 : Capitole, Caton, Charles-Peguy, Festival, Frandoc, Marius, Nabuco, Ouest, Rafa, Reso, Rivoli, Splendeur, Tango, Tenor, Topaze, Valmy.

V ; 5, 7, 10: Aiglon, Aquillon, Aube, Bocquiau, Brennus, Castan, Fanion, Faust, Fortin, Garant, Lutin, Onyx, Petrel, Poncheau.

VI ; 5, 7, 8, 10 : Abo, Aboukir, Adam, Alto, Choisel, Eylau, Favori, Fidel, Floress, Glanor, Languedoc, Lodi, Lutece, Major, Markus, Protinal, Roisel.

VII ; 5, 7, 9, 10 : Apexal, Cledor, Gelpa, Magdalena, Merit, Primo, Wimax.

VIII ; 5, 6, 8, 10 : Bogrin, Carat, Eloi, Flèche d'Or, Goya, Pilote, Rallye, Wattines.

IX ; 4, 7, 8, 12 : Arcole, Champlein, Fleurus, Flųto, Gala, Iena, Jano, Luron, Nicam, Proqual, Rigoudi, Valdor, Yga.

$\mathrm{X} ; 4,7,9,12$ : Alex, Allegro, Talent, Vaillant.

$\mathrm{XI} ; 1,2,7,12$ : Alba, Artois, Capest, Capnord, Copain, Extra, Hybride de la Noue, Manella, Marignan, Marival, Marne, Vague d'Epis, Wim.

XII ; 1, 2, 7, 8, 12 : Amigo, Comtal, Match, Monjoie, Rial.

XIII ; 1, 2, 7, 11 : Cordial, Flinor, Marly.

XIV ; $1,2,7,8,11$ : Bleroy, Elite.

$\mathrm{XV} ; 2,7,8,11:$ Abel.

XVI ; 1, 2, 6, 8, 12 : Avalon, Nautica.

XVII ; 3, 7, 12 : Atou, Fertyl, Frigor, Hobbit, Vilmorin 27.

XVIII ; 3, 7, 8, 12 : Top.

$\mathrm{XiX} ; 3,6,8,12$ : Corin, Diam

$\mathrm{XX} ; 2^{*}, 5,7,8,10$ : Aubaine, Cargo.

$\mathrm{XXI} ; 2^{*}, 5,7,9,10$ : Florence Aurore, Tarasque.

XXII ; 2*, 3, 7, 12 : Camp Remy.

XXIII ; 3, 20, 12 : Vilmorin 53.

XXIV ; $2,2 *, 7,12$ : Hardi.

XXXI $; 1,3,7,12:$ Pia.

XXV ; $2,2^{*}, 7,8,12$ : Courtot, Messidor.

XXVI ; 2, 2*, 17, 18, 12 : Prinqual.

XXVII ; 2, 20, 12 : Maître Pierre, Primepi.

XXVIII ; 4, 7, 12 : Boulmiche, Vilmorin 23.

XXIX ; 5, 20, 10 : Elysee, Gilma, Miche.

XXXII ; 1, 3, 20, $12:$ Noralter.

XXXIII ; $1,4,7,12$ : Orepi.

XXXIV ; 1, 4, 7, 8, 12 : Florent, Dragon, Hybride de Bersée.

XXXV ; $1,5,6,8,10$ : Disponent.

XXXVI ; $1,5,7,10$ : Ducat.

XXX $; 5,14,19,10$ : Prestige. 
(2-7-12). Ainsi les ensembles de diagrammes les plus fréquents sont les suivants : $25 \mathrm{~d}$. (2-7-12), $17 \mathrm{~d}$. (2-7-8-12), 17 d. (5-7-8-10), 16 d. (2-7-9-12), 14 d. (5-7-10), 13 d. (4-7-8-12), 12 d. (2-6-8-12). Lorsqu'on analyse une collection mondiale, on constate que les types 2-7-12, 2-7-8-12 ou 2-7-9-12 se retrouvent moins fréquemment dans les génotypes d'origine étrangère. Ces 3 diagrammes, qui sont au total présents chez près de 30 p. 100 des cultivars, caractérisent assez bien les blés français. Le fait que plusieurs blés aient le même diagramme des gluténines est, dans de nombreux cas, expliqué par leur apparentement.

Il existe néanmoins une certaine diversité dans ces 195 blés. Ce tableau nous révèle en effet que l'on a 13 diagrammes qui ne se rencontrent que chez un seul blé. Dans ce cas le diagramme est pour l'instant caractéristique du génotype. Nous avons également 12 diagrammes différents qui se retrouvent chez 2 ou 3 cultivars seulement. Notons en outre que, pour les 126 blés présents au Catalogue français en 1982 et 1983, nous observons 34 types de diagrammes différents. Dans ceux-ci les 22 blés de printemps se répartissent en 12 types de diagrammes, ce qui constitue une large dispersion relativement à celle des 104 blés d'hiver qui ne donne que 26 types différents. Cette constatation confirme celle réalisée par AUTRAN \& BOURDET (1975) à partir de l'analyse des gliadines et peut s'expliquer par le fait que les blés d'origine étrangère ont été très souvent utilisés dans la création des blés de printemps en France.

\section{Cas des blés durs}

Sur les 38 blés durs analysés nous dénombrons 9 types de diagrammes différents. Comparativement aux blés tendres, les blés durs ne semblent guère plus diversifiés : 9 types de diagrammes pour 38 blés durs contre 38 types pour 195 blés tendres. En fait cette diversité n'est qu'apparente (voir tabl. 3). Le diagramme des gluténines $6-8-10^{\prime}$ et 20 se retrouve respectivement chez 14 et 15 blés durs. Plus des trois

\section{TABLEAU 3}

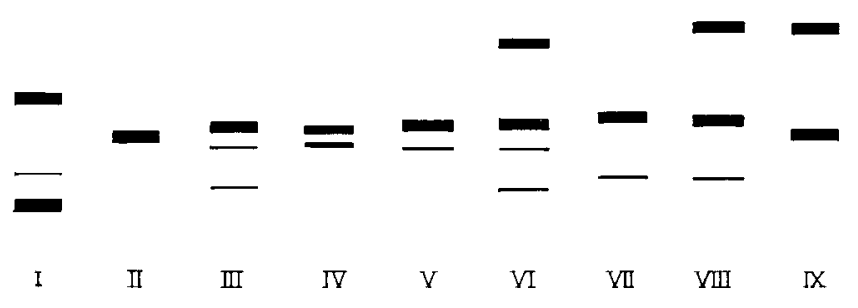

Blés durs.

I ; 6, 8, 10' : Agathe, Brumaire, Calvinor, Cando, Chandur, Flodur, Kidur, Mondur, Montferrier, Poinville, Regal, Valdur, Lakota, Randur.

II ; 20 : Amidur, Bidi 17, Blondur, Casoar, Clairdoc, Diabolo, Durox, Tomclair, Dural, Leeds, Mandon, Telez 3, Viprior, Wells.

III ; 13, 16, 9 : Arcour, Rikita.

IV ; 14, 19 : Romeo, Durandal.

V; 13,16 : Capdur.

VI ; 2*, 13, 16, 9 : Durtal.

VII ; 7, 8 : Cocorit.

VIII ; $1,7,8$ : Mitschsurinka.

IX ; 1, 20 : Skopje. quarts des blés durs ont l'un ou l'autre de ces diagrammes.

Si le seul diagramme des gluténines de haut poids moléculaire est très insuffisant pour identifier un cultivar donné, nous allons voir en revanche que l'analyse de ces protéines comme complément à l'électrophorèse des gliadines peut s'avérer très intéressante. Nous savons en effet que sur l'ensemble des cultivars français, on ne dénombre actuellement pas moins de 60 blés tendres répartis en 19 groupes de 2 à 8 génotypes non différenciables par l'électrophorèse des gliadines. Pour les blés durs, 22 d'entre eux, répartis en 7 groupes de 2 à 9 génotypes, ne peuvent être identifiés avec certitude par la seule lecture du diagramme des gliadines. L'électrophorèse des gluténines de ces cultivars, jusqu'ici non différenciés, permet en effet de distinguer la majorité d'entre eux. Au tableau 4 nous donnons le polymorphisme des gluténines des principaux blés tendres et durs qui formaient ces groupes. Ainsi un bon nombre des blés qui composaient ces groupes sont désormais parfaitement identifiables. Cette identification, qui s'appuie sur l'observation de la diversité des gliadines et des sousunités gluténines de haut poids moléculaire, permet de lever les ambiguités qui subsistaient jusqu'ici dans les transactions des cultivars non distinguables.

Sur le plan de la technique d'identification remarquons qu'il n'est pas nécessaire d'avoir recours chaque fois à l'électrophorèse des gluténines. Dans de nombreux cas l'analyse des gliadines suffit. Lorsqu'il y a ambiguitté il convient alors de procéder à l'extraction des gluténines sur les mêmes culots d'où ont été extraites les gliadines des diagrammes non distinguables. Cette opération exige bien entendu de garder tous les culots d'extraction jusqu'à la révélation du polymorphisme des gliadines.

\section{La diversité des blés appréciée par le polymor- phisme des gluténines}

Avant d'évaluer la diversité des blés à l'aide du polymorphisme des gluténines, il est souhaitable de préciser si la diversité des diagrammes est peu ou non différente de celle attendue en théorie. Compte tenu du déterminisme génétique de ces protéines, on peut en effet évaluer le nombre théorique minimum de diagrammes observables. L'hérédité de ces gluténines précisée par PAYNe et al. $(1980,1982)$ est assez simple. Selon les cultivars, il existe un locus ou deux loci proches l'un de l'autre et peu éloignés du centromère sur chacun des bras longs des chromosomes 1B et 1D. Le bras long du chromosome 1A ne possède qu'un locus codant pour les gluténines. Pour ces différents gènes il existe un multi-allélisme. Ainsi pour les 24 bandes connues jusqu'ici nous avons plusieurs types de polymorphisme : 3 pour $1 \mathrm{AL}, 48$ pour $1 \mathrm{BL}, 15$ pour $1 \mathrm{DL}$, soit au total 2160 types de diagrammes différents en admettant que les loci liés puissent être dissociés. Pour les 18 bandes rencontrées chez les 195 blés français on devrait, sous cette même condition, dénombrer 648 types de diagrammes différents. Si l'on considère maintenant que ces linkats ne peuvent être rompus, avec les 18 bandes des blés français l'on devrait dénombrer $3(1 \mathrm{AL}) \times 7(1 \mathrm{BL}) \times 5(1 \mathrm{DL})=105$ diagrammes différents. Ce nombre de 105 diagrammes 
TABLEAU 4

Polymorphisme des gluténines des groupes de blés non distinguables par électrophorèse des gliadines sur gel d'amidon. Glutenin polymorphism of wheat cultivar groups which cannot be distinguished by starch gel electrophoresis of gliadin.

\begin{tabular}{|c|c|c|c|}
\hline \multicolumn{2}{|c|}{ BLES TENDRES } & $\begin{array}{l}\text { Hardi } \\
\text { Axel } \\
\text { Camp Remy } \\
\text { Diam } \\
\text { Favori } \\
\left.\begin{array}{l}\text { Fleurus } \\
\text { Nicam } \\
\text { Champi }\end{array}\right\} \\
\end{array}$ & \multirow{4}{*}{$\begin{array}{l}2-2 *-7-12 \\
2-7-8-12 \\
2 *-3-7-12 \\
3-6-8-12\end{array}$} \\
\hline \multirow{2}{*}{$\begin{array}{l}\text { Faust } \\
\text { Gaillard }\end{array}$} & \multirow{2}{*}{$\begin{array}{l}5-7-10 \\
2-7-8-12\end{array}$} & \multirow{6}{*}{$\left.\begin{array}{l}\text { Hardi } \\
\text { Axel } \\
\text { Camp Remy } \\
\text { Diam } \\
\text { Favori } \\
\text { Fleurus } \\
\begin{array}{l}\text { Nicam } \\
\text { Champi }\end{array}\end{array}\right\}$} & \\
\hline & & & \\
\hline Top & $3-7-8-12$ & & \\
\hline Noroit & $2-7-12$ & & $5-7-8-10$ \\
\hline \multirow{2}{*}{$\begin{array}{l}\text { Beauchamp } \\
\text { Rafa }\end{array}$} & \multirow{2}{*}{$\begin{array}{l}2-6-8-12 \\
2-7-9-12\end{array}$} & & $4-7-8-12$ \\
\hline & & & $2-7-12$ \\
\hline Heima & $2-6-8-12$ & \multirow{6}{*}{$\left.\begin{array}{l}\text { Ducat } \\
\text { Allegro } \\
\text { Blason } \\
\text { Moisson } \\
\text { Capitole } \\
\text { Festival } \\
\text { Splendeur } \\
\text { Tango }\end{array}\right\}$} & \multirow{3}{*}{$\begin{array}{l}1-5-7-10 \\
4-7-9-12 \\
2-7-8-12\end{array}$} \\
\hline Roazon & $2-7-12$ & & \\
\hline \multirow{2}{*}{$\begin{array}{l}\text { Copain } \\
\text { Merit }\end{array}$} & $1-2-7-12$ & & \\
\hline & $5-7-9-10$ & & $2-7-8-12$ \\
\hline \multirow{2}{*}{$\begin{array}{l}\text { Frandoc } \\
\text { Festin }\end{array}$} & $2-7-9-12$ & & \multirow[t]{2}{*}{$2-7-9-12$} \\
\hline & $2-7-12$ & & \\
\hline Adam & $5-7-8-10$ & \multirow{4}{*}{$\begin{array}{l}\text { Joss } \\
\text { Hamilcar } \\
\text { Palmares } \\
\text { Ramses } \\
\text { Albatros }\end{array}$} & \multirow{2}{*}{$2-6-8-12$} \\
\hline Comtal & $1-2-7-8-12$ & & \\
\hline \multirow{3}{*}{$\left.\begin{array}{l}\text { Caton } \\
\text { Nabuco } \\
\text { Talent } \\
\text { Vaillant }\end{array}\right\}$} & \multirow{2}{*}{$2-7-9-12$} & & \multirow[b]{2}{*}{$2-7-8-12$} \\
\hline & & & \\
\hline & $4-7-9-12$ & \multicolumn{2}{|c|}{ BLÉS DURS } \\
\hline \multirow{6}{*}{$\begin{array}{l}\text { Champlein } \\
\text { Aiglon } \\
\text { Atou } \\
\text { Cocagne } \\
\text { Glanor } \\
\text { Fleuron }\end{array}$} & \multirow{6}{*}{$\begin{array}{l}4-7-8-12 \\
5-7-10 \\
3-7-12 \\
2-6-8-12 \\
5-7-8-10\end{array}$} & \multirow[t]{2}{*}{$\begin{array}{l}\text { Valdur } \\
\text { Blondur }\end{array}$} & \multirow{2}{*}{$\begin{array}{l}6-8-10^{\prime} \\
20\end{array}$} \\
\hline & & & \\
\hline & & \multirow{2}{*}{$\begin{array}{l}\text { Agathe } \\
\text { Capdur }\end{array}$} & \multirow{2}{*}{$\begin{array}{l}6-8-10^{\prime} \\
13-16-9 \\
\end{array}$} \\
\hline & & & \\
\hline & & Lakota & $6-8-10^{\prime}$ \\
\hline & & Wells & 20 \\
\hline \multirow{7}{*}{$\begin{array}{l}\text { Marengo } \\
\text { Gamin }\end{array}$} & $2-7-8-12$ & Calvinor & \multirow{5}{*}{$6-8-10^{\prime}$} \\
\hline & $1-4-7-8-12$ & Chandur & \\
\hline & & Kidur & \\
\hline & & Poinville & \\
\hline & & Regal & \\
\hline & & $\begin{array}{l}\text { Durtal } \\
\text { Durox }\end{array}$ & $\begin{array}{l}2 *-13-16-9 \\
20\end{array}$ \\
\hline & & Rikita & $13-16$ \\
\hline
\end{tabular}

différents est le minimum théorique que l'on peut obtenir avec les 18 bandes observées chez les 195 blés. Or 38 seulement ont été répertoriés dans l'ensemble des blés français. Plusieurs hypothèses peuvent être avancées pour expliquer cette diversité relativement faible des diagrammes des gluténines des blés :

1) la plupart des cultivars seraient issus de croisement entre parents dont les diagrammes sont peu différents voire identiques entre eux. Les possibilités d'obtention de diagrammes de types nouveaux seraient alors très limitées ;

2) la sélection dans les descendances limiterait le nombre des diagrammes.

Cette $2^{\mathrm{e}}$ hypothèse n'est pas invraisemblable car on sait que plusieurs de ces bandes sont corrélées à la qualité technologique des blés. Or, si cette hypothèse était prépondérante sur l'hypothèse 1 , on devrait avoir un grand nombre de blés ayant le diagramme caractéristique d'une bonne qualité technologique, ce qui n'est pas le cas. Plusieurs des bandes qui sont corrélées aux caractéristiques rhéologiques des blés (BRAN-
LARD \& DARDEVET, 1985) sont présentes ici à une fréquence relativement faible.

Ainsi la diversité des diagrammes des blés français est très inférieure à celle attendue. Mais les fréquences des gluténines des blés français sont-elles différentes de celles que l'on observerait dans une collection mondiale ?

Au tableau 5 sont rapportées les fréquences observées par PAYNE et al. (1981b) sur une collection mondiale de 185 cultivars et celles calculées sur les 195 blés. Nous constatons une différence significative entre les fréquences calculées chez les blés français et celles observées sur une collection mondiale. Ces différences sont particulièrement marquées pour les 3 allèles situés sur $1 \mathrm{AL}$. L'absence des bandes 1 ou $2 *$ codées par le chromosome $1 \mathrm{~A}$ caractérise près de 90 p. 100 des blés français. Or la bande 1 a été repérée par PAYNE comme ayant un rôle important dans la qualité des blés. Au sein d'une collection très diversifiée génétiquement et présentant une très grande variabilité de qualité, BRANLARD \& DARDEVET (1985) observent que la bande $2^{*}$ joue un rôle plus important 
TABLEAU 5

Comparaison des fréquences des allèles codant pour les sous-unités gluténines entre une collection mondiale (PAYNE et al., 1981b) et 195 blés cultivés en France.

Comparison of frequencies of the alleles coding for glutenin subunits between a world collection of wheats (PAYNE et al., 1981b) and 195 French cultivars.

\begin{tabular}{|c|c|c|c|c|c|c|c|c|c|c|c|c|c|c|c|c|c|}
\hline Chromosomes & \multicolumn{3}{|c|}{$1 \mathrm{AL}$} & \multicolumn{9}{|c|}{$1 \mathrm{BL}$} & \multicolumn{5}{|c|}{$1 \mathrm{DL}$} \\
\hline Allèles & 1 & $2^{*}$ & Nul & 7 & $\frac{7}{8}$ & $\frac{7}{9}$ & $\frac{6}{8}$ & 20 & $\frac{13}{16}$ & $\frac{13}{19}$ & $\frac{14}{15}$ & $\frac{17}{18}$ & $\frac{2}{12}$ & $\frac{2}{11}$ & $\frac{3}{12}$ & $\frac{4}{12}$ & $\frac{5}{10}$ \\
\hline $\begin{array}{l}\text { Distribution I } \\
\text { (PAYNE } e t \text { al., } \\
1981)\end{array}$ & 28 & 28 & 44 & 19 & 20 & 27 & 19 & 5 & 1,5 & 0,5 & 1,5 & 6,5 & 55 & 0 & 2 & 6 & 37 \\
\hline Distribution II & 16 & 5 & 89 & 33,5 & 32 & 16 & 13 & 4 & 0 & 0,5 & 0 & 1 & 47 & 1 & 7 & 12 & 33 \\
\hline & \multicolumn{3}{|c|}{$\chi^{2}=70,05$} & \multicolumn{9}{|c|}{$\chi^{2}=32,49$} & \multicolumn{5}{|c|}{$\chi^{2}=20,09$} \\
\hline
\end{tabular}

encore que celui de la bande 1 . Ainsi la sélection pour la qualité, si elle avait été effective, aurait dû augmenter en particulier les fréquences de ces bandes 1 et $2 *$. Comme cela n'a pas été le cas, on peut donc avancer que la restriction de la variabilité des types de diagrammes n'est pas ou peu liée à la sélection pour la qualité. Ce faible niveau de variabilité de types de diagrammes apparaît plus attribuable, d'une part, à la proximité génétique des parents entrant dans les croisements et d'autre part, au fait qu'un petit nombre d'entre eux sont très souvent repris comme géniteurs. Cela semble avoir eu égadement comme conséquence d'aboutir à des fréquences alléliques notablement éloignées de celles que l'on rencontre dans une collection mondiale.

Ce polymorphisme des gluténines peut être utilisé pour calculer une distance entre génotypes. La distance entre 2 cultivars est nulle s'ils ont le même diagramme. L'utilisation de ces marqueurs biochimiques pour estimer une distance nous permet de suivre l'évolution de la diversité des blés au fur et à mesure qu'ils apparaissent au Catalogue officiel des espèces et variétés. On calcule pour cela la moyenne et l'écart-type des distances des blés apparus au cours d'une période avec ceux les ayant précédés. La figure 4 représente l'évolution de ces moyennes et des écarts-types des distances pour l'ensemble des 195 blés tendres. On constate une décroissance des distances entre cultivars au cours du temps. La diversité des blés évolue au cours du temps de la même façon lorsqu'elle est calculée à partir du caractère présence/absence des bandes (courbes I) ou de leur structure allélique (courbe II). Dans ce dernier cas, la diversité calculée à partir des 15 allèles qui sont responsables de la synthèse des 18 bandes observées correspond à une distance génétique. Ces courbes confirment les résultats observés par BRANLARD \& CHEVALET (1984) qui notaient une décroissance de la diversité des blés appréciés par: 1) le coefficient de parenté, 2) un ensemble de 26 caractères agronomiques, 3) le polymorphisme des gliadines.

Notons que l'on observe là aussi un maximum de diversité se situant vers 1960 . Ce maximum s'explique essentiellement par l'apparition de cultivars qui possèdent des bandes très rares et qui sont différents entre eux. Parmi ceux-ci citons «Fertyl» (3, 7, 12), «Gilma » $(5,20,10)$ et «Prestige » $(5,14,19,10)$. La précédente étude de diversité comportait 78 blés inscrits entre 1945 et 1978 . Celle rapportée ici en com-

(I) Distance moyenne inter

(II) - - Ecort type inter

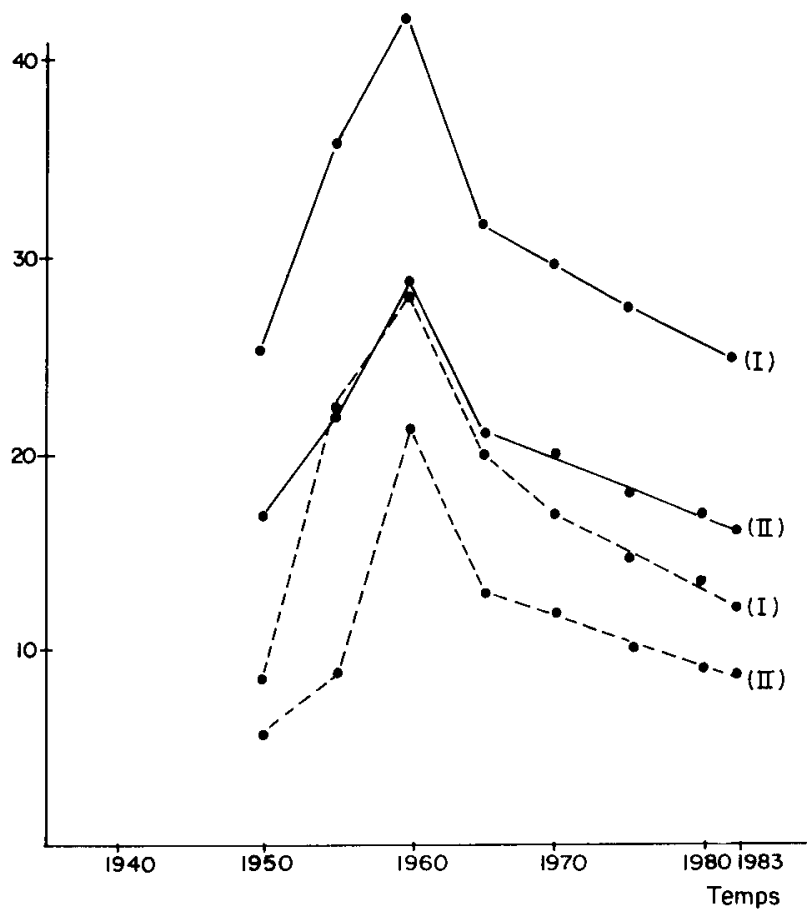

Figure 4

Evolution des moyennes (-) et des écarts-types (--) des distances de blés inscrits depuis 1909. Les blés inscrits au cours des périodes successives de 5 ans sont comparés à l'ensemble des blés les ayant précédés. Distances calculées à partir du caractère présence/absence des bandes (I) ou de leur structure allélique (II).

Change in the mean (-) and standard deviations (--) of distances of wheats registered since 1909 ; wheats registered during a 5-years period were compared with preceding wheats. The distances are calculated: (I) from the presence/absence character of the bands, (II) from their allelic structure. 
prend 195 enregistrés entre 1909 et 1983 . Notons que la décroissance observée jusqu'en 1978, loin de s'inverser ou de se stabiliser, se poursuit jusqu'en 1983. Les principales causes de la diminution des distances entre les cultivars français ont été proposées (BRANLARD \& CHEVALET, 1984). Les résultats présentés ici constituent une confirmation et soulignent l'importance d'introduire et de prendre en compte la diversité génétique dans les programmes et méthodes de sélection.

\section{CONCLUSION}

L'analyse électrophorétique des sous-unités gluténines de haut poids moléculaire des blés tendres français nous a permis de dénombrer 18 bandes différentes. Une bande nouvelle a été décelée ce qui porte à 24 le nombre de sous-unités gluténines de haut poids moléculaire connues à ce jour. Les diverses combinaisons de ces bandes ont donné 38 types de diagrammes, ce qui est peu par rapport à la diversité potentielle pouvant être obtenue des 5 gènes codant pour ces protéines. Sur les 38 blés durs analysés nous n'avons observé que 9 types de diagrammes pour 14 bandes différentes rencontrées. La diversité des blés durs, appréciée par ce polymorphisme, n'est pas supérieure à celle des blés tendres. Pour ces derniers, les calculs de distances sur les gluténines nous permettent de con- firmer, qu'au fur et à mesure de leur inscription au Catalogue officiel, ils apparaissent de plus en plus ressemblants entre eux. Une étude semblable mériterait d'être conduite sur les blés durs avec, bien entendu, une gamme plus étendue de cultivars. L'observation de ces protéines s'est avérée de plus très intéressante pour réaliser l'identification des variétés non distinguables par l'électrophorèse de leurs gliadines sur gel d'amidon. Près de 80 p. 100 des blés non distingués le sont maintenant par l'observation de leurs gluténines. Ces protéines méritent d'être étudiées également pour le rôle qu'elles jouent dans la qualité technologique des blés. Une meilleure connaissance de l'effet de leur présence ou de leur absence sur la structure et les propriétés du complexe glutineux formé avec les gliadines, elles-mêmes beaucoup plus polymorphes, devrait permettre d'accroître l'efficacité de la sélection pour la qualité des blés.

Recu le 13 février 1984 Accepté le 16 janvier 1985.

\section{REMERCIEMENTS}

Les auteurs remercient Mireille DARDEvET pour son aide dans les analyses électrophorétiques et sont très reconnaissants envers J. C. Autran et P. Vincourt (I.N.R.A., Montpellier) pour les remarques et suggestions qu'ils ont portées sur le manuscrit.

\section{RÉFÉRENCES BIBLIOGRAPHIQUES}

Autran J. C., 1981. Recent data on the biochemical basis of durum wheat quality, 257-273. In Charalambous G. \& Inglett G. : «The quality of foods and beverages », vol. I. Acad. Press, 443 p.

Autran J. C., Bourdet A., 1975. L'identification des variétés de blé : établissement d'un tableau général de détermination fondé sur le diagramme électrophérique des gliadines du grain. Ann. Amélior. Plantes, 25, 3, 277-301.

Autrun J. C., Damidaux R., Jeanjean M. F., 1982. Bestimmung des genetisch-bedingten «Teigwaren - Koch potentials » von Durumweizensorten anhand von Electropherogrammen von Glutenproteinen. Getreide Meht und Brot, 36, 29-35.

Autran J. C., Berrier R., Jeanjean M. F., Joudrier P., Kobrehel K., 1981. Emploi de l'électrophorèse dans la filière céréale ; possibilités et limites actuelles. Tech. Ind. Céréales, 8, 3-19.

Branlard G., 1983. Study of genetic determination of 20 gliadin bands. Theor. Appl. Genet., 64, 155-162.

Branlard G., Bellot P., 1983. Improvement of bread wheat gluten quality. Qual. Plant., 33, 121-126.

Branlard G., Chevalet C., 1984. Sur la diversité des blés tendres cultivés en France. Agronomie, 4 (10), 933-938.

Branlard G., Dardevet M., 1985. Diversity of grain protein and bread wheat quality. II. - Correlation between High Molecular Weight glutenin subunits and flour quality characteristics. J. Cereal. Sci. - Accepté pour publication.

Branlard G., Rousset M., 1980. Les caractéristiques électrophorétiques des gliadines et la valeur en panification du blé tendre. Ann. Amélior. Plantes, 30 (2), 133-149.

Branlard G., Triboi E., 1983. Influence du milieu de culture sur les concentrations relatives des bandes des gliadines chez le blé tendre. C.R. Acad. Sci., Paris, 297, IV, 229-232.
Burnouf T., Bouriquet R., 1980. Glutenin subunits of genetically related hexaploid wheat cultivars : their relation to bread-making quality. Theor. Appl. Genet., 58, 107-111.

Damidaux R., Feillet P., 1978. Relation entre les propriétés viscoélastiques du gluten cuit, la teneur en protéines et la qualité culinaire des blés durs. Ann. Technol. Agric., 28, 799-808.

Feillet P., 1965. Contribution à l'étude des protéines du blé : influence des facteurs génétiques, agronomiques et technologiques. Ann. Technol. Agric., 14, HS, 1-94.

Feillet P., Bourdet A., 1967. Composition protéique et caractéristiques génétiques des blés. Bull. Soc. Chim. Biol., 49, 1273-1283.

Kasarda D. P., Bernardin J. E., Qualset C. O., 1976. Relationship of gliadin protein components to chromosomes in hexaploid wheat (Triticum aestivum L.). Proc. Natl. Acad. Sci. USA, 73, 3645-3650.

Laemmli U. K., 1970. Cleavage of structural proteins during the assembly of the head of bacteriophage T4. Nature, 227, 680-685.

Le Blanc A., 1984. Etude de la qualité des blés tendres par l'analyse électrophorétique des gluténines. Intérêt pour la sélection. Thèse Doct. Ing. Univ. Lille, $n^{\circ} 362,209$ p.

Moonen J. H. E., Scheepstra A., Graveland A., 1982. Use of the SDS sedimentation test and SDS polycrylamide gel electrophoresis for screening breeder's sample of wheat for bread making quality. Euphytica, 31, 677-690.

Mosse J., 1968. Les protéines des céréales, 47-81. In Lavollay J. : "Progrès en chimie agricole et alimentaire », Hermann Ed., Paris, $115 \mathrm{p}$.

Payne P. I., Lawrence G. J., 1983. Catalogue of alleles for the complex gene loci, Glu-A1, Glu-B1 and Glu-D1 which code for high-molecular-weight subunits of glutenin in hexaploid wheat. Cereal Res. Com., 11 (1), 29-33. 
Payne P. I., Corfield K. G., Blackman J. A., 1979. Identification of a high-molecular weight subunit of glutenin whose presence correlates with bread-making quality, in wheats of related pedigree. Theor. Appl. Genet., 55, 153-159.

Payne P. I., Corfield K. G., Holt L. M., Blackman J. A., 1981a. Correlations between the inheritance of certain high molecular weight subunits of glutenin and bread-making quality in progenies of six crosses of bread wheat. J. Sci. Food Agric., 32, 51-60.

Payne P. I., Holt L. M., Law C. W., 1981b. Structural and genetical studies on the high molecular weight subunits of wheat glutenin. Theor. Appl. Genet., 60, 229-236.

Payne P. I., Holt L. M., Worland A. J., Law C. N., 1982. Structural and genetical studies on the high-molecular-weight subunits of wheat glutenin. Part 3. Telocentric mapping of the subunit genes on the long arms of the homeologous group 1 chromosomes. Theor. Appl. Genet., 63, 129-138.

Payne P. I., Holt L. M., Lawrence G. J., 1983. Detection of a novel high molecular weight subunit of glutenin in some Japanese hexaploid wheats. J. Cereal Sci., 1, 3-8.

Payne P. I., Law C. N., Mudd E. E., 1980. Control by homoeologous Group 1 chromosomes of the high-molecular weight subunits of glutenin, a major protein of wheat endosperm. Theor. Appl. Genet., 58, 113-120.

Wrigley C. W., Shepherd K. W., 1973. Electrofocusing of grain from wheat genotypes. Ann. N.Y. Acad. Sci., 205, 154-162. 